\title{
Research on the joint training mechanism of graduate students in \\ Collaborative Innovation
}

\author{
Yaqi $\mathrm{Xu}^{1, \mathrm{a}}$,Shidan $\mathrm{He}^{1, \mathrm{~b}}$ \\ ${ }^{1}$ Xijing University,Xian,China,710123 \\ axuyaqi@xijing.edu.cn
}

Key words: Collaborative innovation; postgraduate joint training; Mechanism

\begin{abstract}
Today graduate education as the highest level in higher education plays a more and more important role. China graduate education achievements also exposed some problems, so the research training mode is the key to ensure the quality of postgraduate education. The construction of university high level characteristics and improving the quality of talents at present, cooperative education as one of the cooperative innovation mode, which help give full play to the talent advantage and resource advantage, and improve the comprehensive quality of students. Therefore cooperative education is the effective way to promote the sustainable development of society. This paper analysis the impact of various dynamic factors related subjects involved in joint training, summarized the current postgraduate dynamic mechanism problems about joint training, and put forward the perfect joint training mechanism suggestions.
\end{abstract}

\section{Introduction}

the country officially launched the "plan to enhance the innovative ability of College" in May7, 2012.which plan to "talent, discipline, scientific research" three-in-one enhance innovation ability as the core task, that in order to break through the barrier mechanism of internal and external system in colleges ${ }^{[1]}$, vigorously promote the scientific research institutes, and to promote the full sharing of high-quality resources. The plan is not only to explore collaborative innovation mode to adapt to different needs in scientific research, but also to create an environment and atmosphere. Joint training is an important mode, deepen the reform of higher the education system in our country's top-notch innovative talents that is to promote the education and scientific research organically, improve the ability of independent innovation.

\section{The Factor analysis of dynamic mechanism}

"Dynamic mechanism" is a mode of action that internal and external forces to promote the movement, change, development in the system. The stability of dynamic mechanism can make the overall operation of the system from spontaneity to consciousness. Collaborative innovation environment joint postgraduate training mechanism refers to the government policies and regulations and under the guidance of the concept of collaborative innovation, driven enterprises, universities, research institutes and other core subject in-depth, effectively to carry out joint postgraduate training, all the dynamic factors cultivate top-notch innovative talents, and the power 
structure system formed by these factors and its operation rules. Dynamic mechanism is based on the demands of cooperation ${ }^{[2]}$, composed of various elements, the multi subject participation, and the elements of interaction, mutual restraint, mutual existence and development, is to promote joint postgraduate training to effectively carry out the fundamental strength. Dynamic mechanism exists in the collaborative innovation system of internal motivation which is a dynamic factor fundamental mainly, including strategic coordination guidance, interests driving force, internal incentive force and government support force.

The strategic synergy. Strategic synergy guide function is the main strategic objectives about political research collaborative, and guide the value orientation and behavior of each member orientation in the collaborative innovation system. University hoped that the joint optimization of talent Culture the talent training mode to improve the comprehensive innovation ability, promote cross and emerging disciplines.

The interest drive. The interest drive is the fundamental driving force for promoting joint training, and maximizes their benefits that are to promote joint postgraduate training inexhaustible power to continue in-depth development, combined with the cultivation. The interest drive mainly includes the training funds, management cost, state funded subsidies, ownership, reward, achievement, reputation.

The internal motivations. The internal motivation is that the organizers make the behavior of members and its goals are compatible, and play to the potential of each member. The internal motivation through a series of specific organizational norms behavior to run, which is according to the members of the organization survival and development requirements ${ }^{[3]}$, values of design rewards and punishment system under the environment of collaborative innovation. Joint postgraduate training is a systems engineering, personnel training is mainly from the main behavior within the system to implement, is the main innovation synergy process.

The government support. Government support is an important dynamic for joint postgraduate training indispensable, which encourage and guide the work of joint training to effectively carry out in-depth, and plays an irreplaceable role.

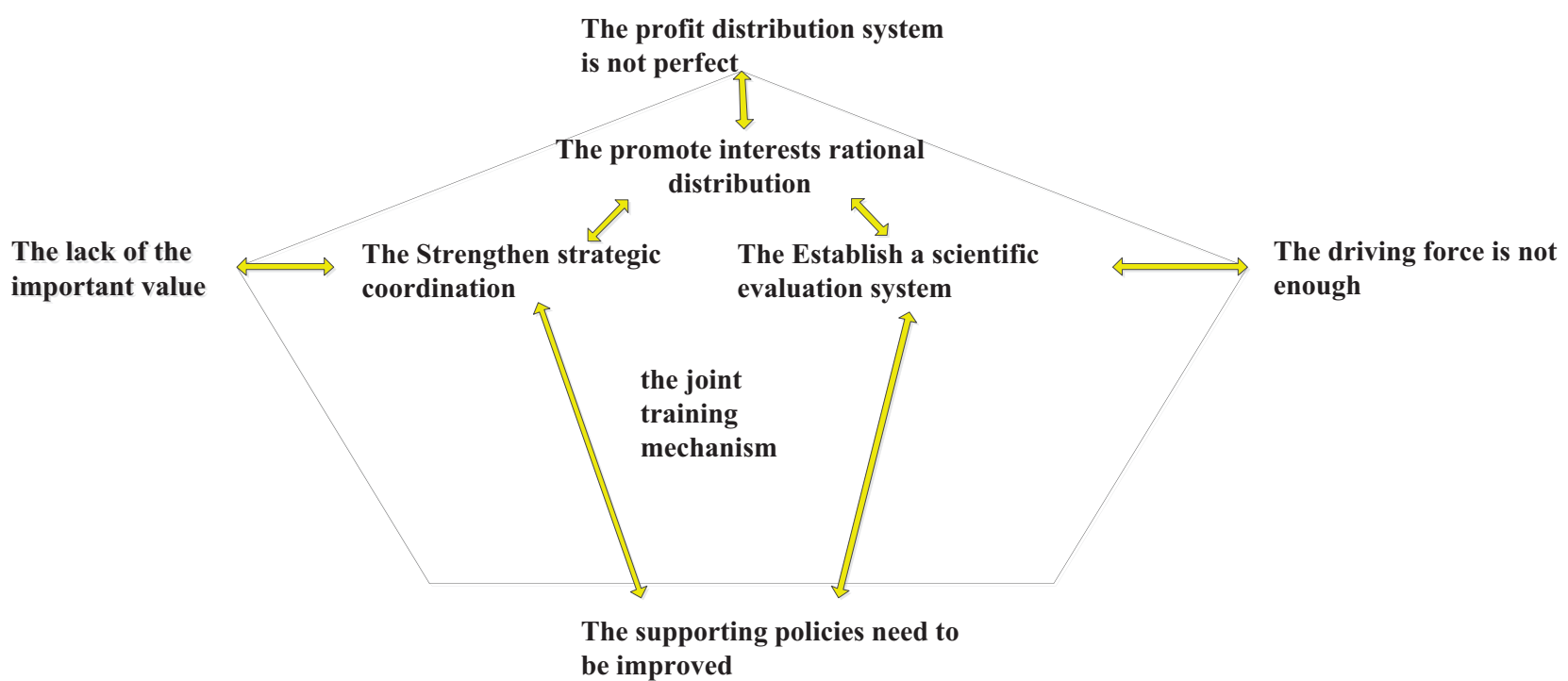

Fig1. The joint training mechanism

\section{The existing problems of joint postgraduate training mechanism}


The joint postgraduate training mechanism is not perfect make the main body lack power, and vitality is not strong. The existing problems of joint postgraduate training mechanism as following aspects:

The lack of the important value .we put out the 《national long-term education reform and development plan (2010 2020)》In order to enhance the innovation ability of higher education, improve the quality of higher education, training innovative talents, building an innovative country. The new mechanism makes each subject of thinking, values, concept is different, and it is difficult to joint each other's values and culture. The formation of different or even is opposite potential organizational culture and behavior criterion. At the same time the lack of joint training form a strong resonance, which is not carrying out development strategy, and result the implementation of the joint training work. These have greatly restricted the participation in the joint training.

The profit distribution system is not perfect. The joint training is mainly related to training funds, management fees, the state funded subsidies and other material benefits, the patent ownership, reward, reputation, and other non-material interests. The benefit distribution is easy to appear the following problems: first, the distribution of material benefits is easy to appear that training division of funds is not clear. Secondly, the participation and cooperation of the units and individuals are so mush, which is easy to appear the actual definition of each person's contribution to the deviation and contradiction. A variety of reasons lead to the interests of the distribution system is not perfect [4], participation is difficult to improve in joint training of graduate students, also restricts the initiative.

The driving force is not enough. The standard differences often make joint training work is difficult to carry out, and the lack of an effective incentive mechanism can greatly reduce the subjects of the top-notch innovative personnel training enthusiasm. universities and research institutes make research papers, patents, and access levels and quantity of awards as evaluation standards, not paid much attention to application of patent, scientific research, ignoring the instructors in training graduate students creativity, cultural heritage and other aspects of the performance and reputation, do not attach importance to theoretical teaching and professional the joint training of graduate students in the research process .many researchers have dampened the enthusiasm of bowel, carry out joint training creativity, engrossed in their professional fields to do research, write papers. Enterprises pay attention to the graduate knowledge application and technical transformation ability, scientific research value of market prospects and improve their technical level and professional guidance, teaching practice in the enterprise occupation promotion standards work which do not attach importance to enterprises in joint training of Instructors, and ignoring the contribution of cultivating high quality talents with high skill.

The supporting policies need to be improved. The government as the top designers joint training, which want to enhance the advocates of collaborative innovation to lead the innovation, and to develop 《the Ministry of education to carry out joint training of graduate work notice》(Department of 2003 No. 3), 《the development of universities and research institutions, joint training doctoral students working procedures》 (Department of 2009 No. 5), which plans to enhance the innovation capacity of higher school. These documents guide and support the higher schools and all kinds of innovative forces to carry out in-depth cooperation in the institutional framework, which promote the full sharing of high-quality resources, to achieve synchronization, and to enhance the training quality and the ability of scientific research. At the same time, we are due to the lack of promoting the joint training work plan to guide and support, the joint training plan is still relatively simple. Collaborative level is not deep enough, failed to effectively mobilize the enthusiasm and initiative 
of the joint training, give full play to the government guidance on joint the development direction, which is not conducive to the healthy development, coordinate and promote joint training. 


\section{The Countermeasures for joint training of graduate students}

The core of sustainable development about graduate student training is Perfect joint postgraduate training mechanism, stimulating the initiative of each subject collaborative education.

The Strengthen strategic coordination. The strengthen strategic cooperation ask to establish a new concept of joint postgraduate training, which is to strengthen strategic cooperation. The joint training requirements of each subject is fully understand the strategic synergy thought, driving the development strategy of talent strategy, innovation, deep understanding. The talent is the fundamental education that is a new concept. Combined with the cultivation of basic political research Quartet together, communicate and interact fully in personnel training, coordination of each other's cultures and values, improve the strategic project of science and technology innovation ability Standard. The strategic cooperation is conducive to promoting discipline construction in Colleges and universities improve the market competitiveness of talents and comprehensive innovation ability; scientific research institutes to improve the conversion rate enterprises reduce training costs, promote enterprise knowledge innovation. The implementation of regional science and technology innovation capacity, promote regional economic development. the strengthen strategic cooperation will gradually lead to the group behavior, the value orientation and behavior orientation and the strategic objectives of the joint training agreement, fully stimulate the main training enthusiasm and vitality.

The promote interests rational distribution. Joint postgraduate training involves many stakeholders, which the deal state is closely connected to all parties involved in the joint forces training, to enhance the enthusiasm and initiative of all subjects by constructing the interest distribution system perfect. First, Joint postgraduate training can set up a joint postgraduate training special the fund management group, the implementation make clear regulations on the classification in various stages, each subject is based on the voluntary consultation in accordance with the joint training agreement strictly. Secondly, the income distribution system should be established contribution, and interests are closely linked the actual contribution in the distribution of interests. Joint postgraduate training should fully consider various cooperative units made in the production process of the contribution, which can guide the signature according to the actual situation.

The Establish a scientific evaluation system. We can use the core policy and economic means to guide and encourage the universities, research institutes, and enhance China's science and technology innovation ability. The government should strengthen the planning and guidance, which can be to joint training of prospective, feasibility, from the strategic height, joint training strategic development planning at the national level. The concept and research institutes, enterprises and other main innovation are to build joint training bases which can courage scientific research institutes.

\section{Conclusion}

We explore the multi subject integration advantages of resources in dynamic mechanism and training In the collaborative innovation environment, which is an important way to high-quality top-notch innovative talents with innovative spirit. The innovation ability, practice ability is not only training, and improve the ability, but also is the implementation of independent innovation strategy, talent strategy. The China's inherent requirements and key links universities, research institutes, government, enterprise as an important force of high-level personnel training, which make joint training should to take the initiative for break the institutional barriers in the process of 
study, and play resource sharing advantage. We should establish joint training mechanism, effective convergence of various elements, and actively participate in the training.

\section{Acknowledgments}

Xijing University research,project number:XJYJ1611

\section{References}

[1] Chen Jin, Yang silver Juan. Collaborative [J] scientific theory foundation and connotation of innovative research, 2012, (.2): 15-23.

[2] China graduate and postgraduate education development report group. China's degree and graduate education development report: 2011[M]. Beijing: Tsinghua University press, 2012:12-62

[3] and Yubing. Collaborative innovation model [J] .science research, 2012, (.2) 116-121.

[4] Shi Yuexiang, Tao Zhiping. Research on the problem of the innovation ability of graduate [J]. students in the University, industry and information technology, 2013, (10) 4-11. 